\title{
MEDIO SOCIAL Y USO SIMULTÁNEO DE ALCOHOL Y TABACO EN ESTUDIANTES UNIVERSITARIOS DE PREGRADO DE CARRERAS DE CIENCIAS DE LA SALUD DE UNA UNIVERSIDAD, CUNDINAMARCA - COLOMBIA
}

\author{
Mónica Veloza Gómez ${ }^{1}$, Laura Simich ${ }^{2}$, Carol Strike ${ }^{3}$, Bruna Brands ${ }^{4}$, Norman Giesbrecht $^{5}$, Akwatu Khenti ${ }^{6}$
}

\footnotetext{
${ }^{1}$ MSc en Enfermería. Profesora Asistente. Programa de Enfermería. Facultad de Enfermería y Rehabilitación, Universidad de La Sabana. Colombia. Colombia. E-mail: monica.veloza@unisabana.edu.co

2 Ph.D. Researcher, Health Systems Research and Consulting Unit. Centre for Addiction and Mental Health. University of Toronto. Toronto, Canada. E-mail: laura_simich@camh.net

${ }^{3}$ Ph.D. Researcher. Health Systems Research and Consulting Unit. Centre for Addiction and Mental Health, University of Toronto. Toronto, Canada. E-mail: carol_strike@camh.net

${ }^{4}$ Ph.D. Researcher. Office of Research and Surveillance, Drug Strategy and Controlled Substances Program. Health Canada and Public Health and Regulatory Policies. Centre for Addiction and Mental Health. University of Toronto. Toronto, Canada. E-mail: bruna_brands@camh.net

${ }^{5}$ Ph.D. Researcher. Health Systems Research and Consulting Unit. Centre for Addiction and Mental Health. University of Toronto. Canada. E-mail: norman_giebsbrecht@camh.net

${ }^{6}$ MSc. Director of International Office. Center for Addiction and Mental Health, Toronto, Canada. E-mail: akwatu_khenti@ camh.net
}

RESUMEN: Estudio transversal cuyo objetivo fue describir el medio social relacionado con el policonsumo simultáneo de alcohol y tabaco, por parte de los estudiantes universitarios de pregrado de las carreras de ciencias de la salud, en una universidad de Cundinamarca, Colombia. Se aplicó un cuestionario a 451 estudiantes. Resultados: $22.6 \%$ de los estudiantes reportaron usar en forma simultánea alcohol y tabaco. Se identificaron las siguientes situaciones como de mayor prevalencia: el aprendizaje de esta combinación es a través de amigos y se consume predominantemente fuera de la universidad; este se hace para relajarse y disfrutar la compañía de los amigos, controlando así el estrés que generan las tareas universitarias y los conflictos familiares. Consideramos necesario desarrollar programas preventivos de alto impacto para ayudar a esta población universitaria, quienes a futuro estarán a cargo del bienestar de la población en general.

DESCRIPTORES: Estudiantes. Drogas ilicitas. Conducta adictiva. Trastornos relacionados con substancias. Factores de riesgo

\section{SOCIAL ENVIROMENT AND SIMULTANEOUS USE OF ALCOHOL AND TOBACO AMONG UNDERGRADUATE STUDENTS IN HEALTH SCIENCES PROGRAMS FROM ONE UNIVERSITY, CUNDINAMARCA - COLOMBIA}

\begin{abstract}
A transversal study whose aim was to describe the social environment associated with simultaneous polydrug use of alcohol and cigarettes, by the undergraduate students of careers of health sciences, at a university in Cundinamarca, Colombia. A questionnaire was administered to 451 students. Results: $22.6 \%$ of students reported using alcohol simultaneously cigarette aged between 18 and 26 years in the social environment represented by the triad (person-environment-psychoactive substances), identifies the following situations: this combination is learning through friends outside the university, they can relax and enjoy the company of friends, thus controlling the stress of academic tasks, and family conflict, facing conflict in their interpersonal relationships. We need to develop high-impact preventive programs to control a situation of great social cost worldwide.

DESCRIPTORS: Students. Street drugs. Addictive behavior. Substance-related disorders. Risk factors.

\section{AMBIENTE SOCIAL E USO SIMULTÂNEO DE ÁLCOOL E TABACO ENTRE ESTUDANTES UNIVERSITARIOS DOS CURSOS DE GRADUAÇÃO EM CIÊNCIAS DA SAÚDE DE UMA UNIVERSIDAD, CUNDINAMARCA - COLÔMBIA}

RESUMO: Estudo transversal, cujo objetivo foi descrever o ambiente social associado ao uso simultâneo de múltiplas drogas, pelos estudantes de graduação na área de Ciências da Saúde, de uma universidade em Cundinamarca, Colômbia. Um questionário foi aplicado a 451 alunos. Resultados: 22,6\% dos alunos, com idade entre 18 e 26, relataram uso de álcool, simultaneamente com tabaco, no ambiente social, representado pela pessoa (tríade ambiente de substâncias psicoativas). Foram identificadas as seguintes situações: aprendizagem para o uso de drogas através de amigos fora da universidade; entendem que podem relaxar e desfrutar da companhia dos amigos, e assim, controlar o stress das tarefas escolares e de conflitos familiares e em suas relações interpessoais. Conclui-se que é necessário desenvolver alto impacto de programas preventivos para controlar uma situação de grande custo social no mundo inteiro. DESCRITORES: Estudantes. Drogas ilícitas. Comportamento aditivo. Transtornos relacionados ao uso de substancias. Fatores de risco. 


\section{INTRODUCCIÓN}

El uso simultáneo de sustancias psicoactivas, se constituye en una situación problema especialmente para la población joven quien como blanco vulnerable hace parte de un medio social, en donde están presentes variedad de sustancias psicoactivas, así como aspectos de índole personal, familiar y social, propios al joven universitario.

El Informe Mundial sobre las Drogas 2008, elaborado por la Oficina de las Naciones Unidas contra la Droga y el Delito (ONUDD), reportó un incremento en el consumo de drogas en el mundo y especialmente en América Latina. Se estima que 208 millones de personas usan drogas, lo que equivale a un $4.9 \%$ de la población mundial; el rango de edad entre los consumidores se encuentra entre los 15 y 64 años. ${ }^{1}$

Adicionalmente el estudio, "Desaprovechar lo mejor y lo de mayor capacidad: El abuso de sustancias en las universidades americanas", ${ }^{2}$ afirma que la principal droga usada en las universidades es el alcohol (65\%-0\%) y que el fenómeno del abuso de sustancias psicoactivas va mas allá de su uso incluyendo el aumento en el uso de otras drogas lícitas e ilícitas. La tendencia identificada en las últimas décadas se ha intensificado y los estudiantes están inmersos en una cultura de abuso de sustancias adictivas con consecuencias en los ámbitos de la salud, académico y social, involucrando a toda la comunidad universitaria.

Actualmente se presenta un nuevo patrón de uso de sustancias psicoactivas que hace referencia al policonsumo simultáneo; es decir el uso simultáneo de dos o más sustancias psicoactivas. ${ }^{3}$ Uno de los patrones de uso simultáneo identificado con mayor frecuencia, es la combinación de alcohol y tabaco.

Como parte del medio social, el proceso de transición del colegio a la universidad puede constituirse en una experiencia particular. La vida universitaria es con frecuencia una cultura en sí misma, con oportunidades para experimentar el consumo de varias sustancias a las cuales no se había estado expuesto anteriormente. Un ejemplo de ello es el caso de "fiestas" desenfrenadas dentro o fuera de la universidad, y la promoción que se hace del uso del alcohol y otras sustancias para disminuir el estrés y divertirse. ${ }^{4}$

En este proceso de transición que vive el estudiante, el ajuste ante nuevas dinámicas de interacción social, la vivencia de la presión de grupo, el manejo del tiempo libre, así como la facilidad ante el acceso a diferentes sustancias psicoactivas, son aspectos que ahora conforman su medio social y se manifiestan en este estudio a través de variables como el aprendizaje y lugar donde se lleva a cabo el uso simultáneo de alcohol y tabaco, así como las situaciones que generan estrés o ansiedad en el estudiante universitario, las razones de este uso y la experimentación de situaciones particulares relacionadas con el uso simultáneo de estas sustancias psicoactivas.

La Teoría del Aprendizaje Social ${ }^{5}$ y la Teoría Biopsicosocial, ${ }^{6}$ son los referentes analíticos para interpretar este medio social y su relación con el uso simultáneo de alcohol y tabaco; sus bases teóricas describen el comportamiento humano operante dentro de una estructura dinámica conformada por factores personales, comportamientos y eventos medioambientales, así como por las esferas biológica, psicológica y social.

Como referentes analíticos, estas teorías permiten considerar al ser humano en este caso representado por el estudiante universitario de pregrado, como la persona en interacción continua con el entorno, binomio interactuante en donde están presentes en forma paralela determinantes biológicos, psicológicos y socioculturales, así como procesos de interpretación e imaginarios que se conjugan con una perspectiva social de la cual hacen parte la historia personal, familiar y social del joven universitario con relación al fenómeno del uso simultáneo de alcohol y tabaco.

El objetivo fue describir el medio social relacionado con el policonsumo simultáneo de alcohol y tabaco, por parte de los estudiantes universitarios de pregrado de las carreras de ciencias de la salud, en una universidad de Cundinamarca, Colombia.

\section{METODOLOGÍA}

Estudio de tipo transversal ${ }^{7}$ que fue parte de un proyecto multicéntrico. La población objetivo estuvo conformada por estudiantes universitarios de pregrado de las carreras de Medicina, Enfermería y Rehabilitación, que estuvieran cursando a tiempo parcial o completo, alguno de los dos primeros años (I, II, III o IV semestre) de carrera.

Con el fin de que todos los estudiantes tuvieran igual oportunidad de participar en el estudio, se seleccionaron aleatoriamente los cursos 
obligatorios de cada facultad y el año de estudio requerido de los participantes. La participación fue voluntaria, mediante la firma de un consentimiento informado. La muestra se conformó con 451 estudiantes, quienes fueron encuestados durante el primer semestre de 2009.

La recolección de datos se desarrolló mediante el método de encuesta, aplicando un cuestionario anónimo auto-administrado, elaborado por el grupo de investigadores del "Programa internacional para el fomento de la capacidad de investigación en profesionales relacionados con la salud para estudiar el fenómeno de las drogas en América Latina y el Caribe, 2008 - 2009", programa patrocinado por la Comisión Interamericana para el Control del Abuso de Drogas (CICAD) de la Organización de Estados Americanos (OEA) y el Centro de Adicciones y Salud Mental de Canadá (CAMH). El instrumento quedó conformado por cuatro secciones y 58 preguntas cerradas, correspondientes a características sociodemográficas y características relacionadas con el uso de sustancias psicoactivas. El cuestionario fue elaborado en idioma inglés, traducido al español y contra-traducido a su idioma original para incrementar la validez idiomática y contenido semántico. Previo a la aplicación fue analizada su validez facial (de apariencia) y su validez de contenido.

La recolección de datos se realizó en los salones de clase y bajo la coordinación del grupo de profesores investigadores del Grupo CICAD/ OEA de la Institución Universitaria. Se emplearon dos cajas selladas destinadas al depósito de los consentimientos informados y los cuestionarios, garantizando así la seguridad en su manejo y posterior archivo.

Los datos fueron analizados descriptivamente. Para el análisis estadístico se utilizó el programa SPSS, versión 15.

Esta investigación fue aprobada previamente por el Research Ethics Board (REB) del Centre for Addiction and Mental Health (CAMH) (Protocol Reference \#225/2008) y por el Comité de Ética en Investigación de la universidad colombiana participante en el estudio.

\section{RESULTADOS}

\section{Características de la muestra}

En el estudio participaron un total de 451 estudiantes, 347 mujeres y 104 hombres, con edades comprendidas entre los 19 y 26 años.

\section{Uso simultáneo de alcohol y tabaco}

El policonsumo simultáneo de alcohol y tabaco y fue reportado por 102 estudiantes quienes conforman el $22.6 \%$ del total de la muestra. El $19.6 \%$ de las mujeres encuestadas y el $32.7 \%$ de los hombres refirieron consumo simultaneo de estas drogas (datos no mostrado en tablas y figuras).

\section{Aprendizaje y lugar relacionado con el uso simultáneo de alcohol y tabaco}

Respecto al aprendizaje relacionado con el uso simultáneo de alcohol y tabaco, las categorías referidas en orden decreciente fueron: aprendizaje por medio de un amigo $43 \%$, por sí mismo $16 \%$, por medio del novio/a $13 \%$, por medio de un miembro de la familia $12 \%$, por medio de otra persona $10 \%$ y por los medios de comunicación $2 \%$. Respecto al lugar en donde se realiza el uso simultáneo de alcohol y tabaco, refirieron en orden decreciente: fiestas fuera de la universidad/discotecas o clubes nocturnos $87 \%$, fiestas universitarias $34 \%$, sitio de residencia lejano a la universidad $27 \%$, sitio de residencia cercano a la universidad y otros lugares (calle, casa de amigos) 18\% (datos no mostrados en tablas o figuras).

\section{Situaciones principales de estrés o ansiedad y uso simultáneo de alcohol y tabaco}

La figura 1, resume los hallazgos de estas variables. Las siguientes situaciones que generan estrés o ansiedad fueron referidas, con frecuencia decreciente: tareas universitarias, problemas familiares, horario universitario, problemas económicos, problemas en las relaciones de pareja, problemas de trabajo, vida social/amistades, y actividades comunitarias. 
Figura 1 - Situaciones principales de stres o ansiedad y uso simultáneo de alcohol y tabaco, Cundinamarca Colombia, 2009

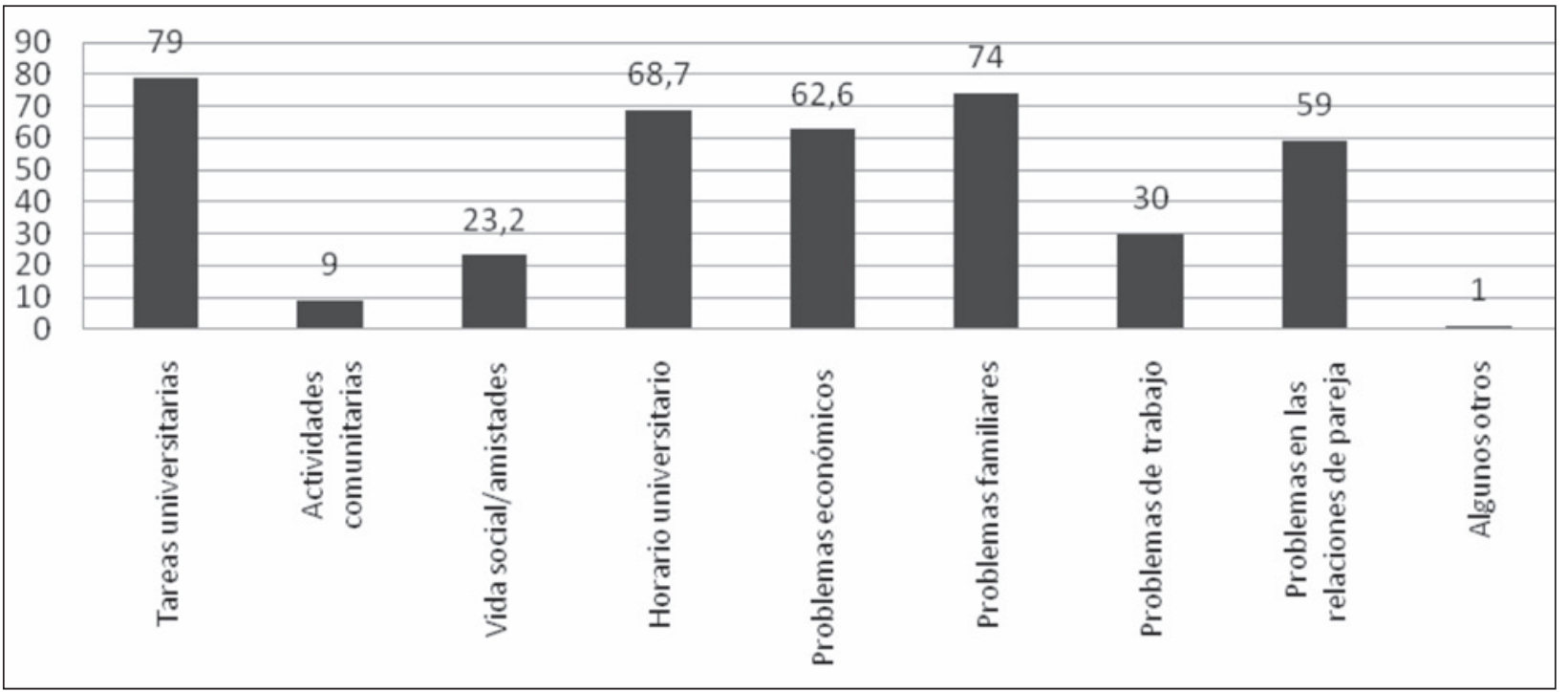

Razones para el uso simultáneo de alcohol y tabaco

La tabla 1 resume los hallazgos de estas variables. En el aspecto central "cambio de ánimo", con mayor porcentaje se destacan las siguientes razones: "le ayudan a relajarse", "le ayudan a dejar de preocuparse por un problema", y "le hacen sentir mejor cuando está deprimido". En el aspecto central "fines sociales", con mayor porcentaje se destacan las siguientes razones: "le ayudan a dis- frutar la compañía de sus amigos" y "le ayudan a mantenerse bien cuando sale en la noche con sus amigos". En el aspecto central "facilitación de actividades", con mayor porcentaje se destaca la siguiente razón: "le ayudan a hacer algo que estaba haciendo, menos aburrido". En el aspecto central "efectos físicos", con mayor porcentaje se destacan las siguientes razones: "le ayudan a permanecer despierto", "mejora las sensaciones cuando tiene sexo", y "le ayuda a perder peso".

Tabla 1- Razones para usar en forma simultánea alcohol y tabaco Cundinamarca-Colombia, 2009

\begin{tabular}{|c|c|c|c|}
\hline \multirow{2}{*}{ Aspecto central } & \multirow{2}{*}{ Razones } & \multicolumn{2}{|c|}{ Frecuencias } \\
\hline & & SI & $\%$ \\
\hline \multirow{5}{*}{ Cambio de ánimo } & Le hacen sentir mejor cuando está deprimido & 57 & 55.9 \\
\hline & Le ayudan a dejar de preocuparse por un problema & 58 & 56.9 \\
\hline & Le ayudan a relajarse & 86 & 84.3 \\
\hline & Le ayudan a sentir emoción o euforia (para experimentar) & 28 & 27.5 \\
\hline & Para sentirse fuertemente drogado o intoxicado & 2 & 2.0 \\
\hline \multirow{4}{*}{ Efectos físicos } & Mejora las sensaciones cuando tiene sexo & 22 & 21.6 \\
\hline & Le ayudan a permanecer despierto & 29 & 28.4 \\
\hline & Le ayudan a perder peso & 13 & 12.7 \\
\hline & Le ayudan a dormir & 11 & 10.8 \\
\hline \multirow{5}{*}{ Fines sociales } & Le ayudan a disfrutar la compañía de sus amigos & 54 & 52.9 \\
\hline & $\begin{array}{l}\text { Le ayudan a sentir más confianza o ser más capaz de hablar con las } \\
\text { personas en una situación social }\end{array}$ & 36 & 35.3 \\
\hline & Le ayudan a perder sus inhibiciones & 34 & 33.3 \\
\hline & Le ayudan a mantenerse bien cuando sale en la noche con sus amigos & 47 & 46.1 \\
\hline & Para relacionarse mejor con los demás debido a la presión social & 16 & 15,7 \\
\hline \multirow{3}{*}{$\begin{array}{l}\text { Facilitación de } \\
\text { actividades }\end{array}$} & Le ayudan a concentrarse en el trabajo o en el estudio & 13 & 12.7 \\
\hline & Mejora las actividades como escuchar música, jugar o practicar un deporte & 12 & 11.8 \\
\hline & Le ayudan a hacer algo que estaba haciendo, menos aburrido & 38 & 37.3 \\
\hline \multirow{2}{*}{$\begin{array}{l}\text { Manejo de los efectos } \\
\text { de otras sustancias }\end{array}$} & Mejora los efectos de otras sustancias & 15 & 14.7 \\
\hline & Le ayudan a atenuar los efectos posteriores de otras sustancias & 8 & 7.8 \\
\hline
\end{tabular}


Vivencia de situaciones particulares y uso simultáneo de alcohol y tabaco

La figura 2 resume los hallazgos de estas variables. Respecto a la vivencia de situaciones particulares relacionadas con el uso simultáneo de alcohol y tabaco, fueron referidas en orden decreciente: conflictos familiares, conflictos con su pareja, dificultades económicas, tener sexo sin protección, tener relaciones sexuales inesperadas/ no planeadas, y ausentarse de clases en forma repetida.

Figura 2 - Situaciones particulares y uso simultáneo de alcohol y tabaco Cundinamarca-Colombia, 2009

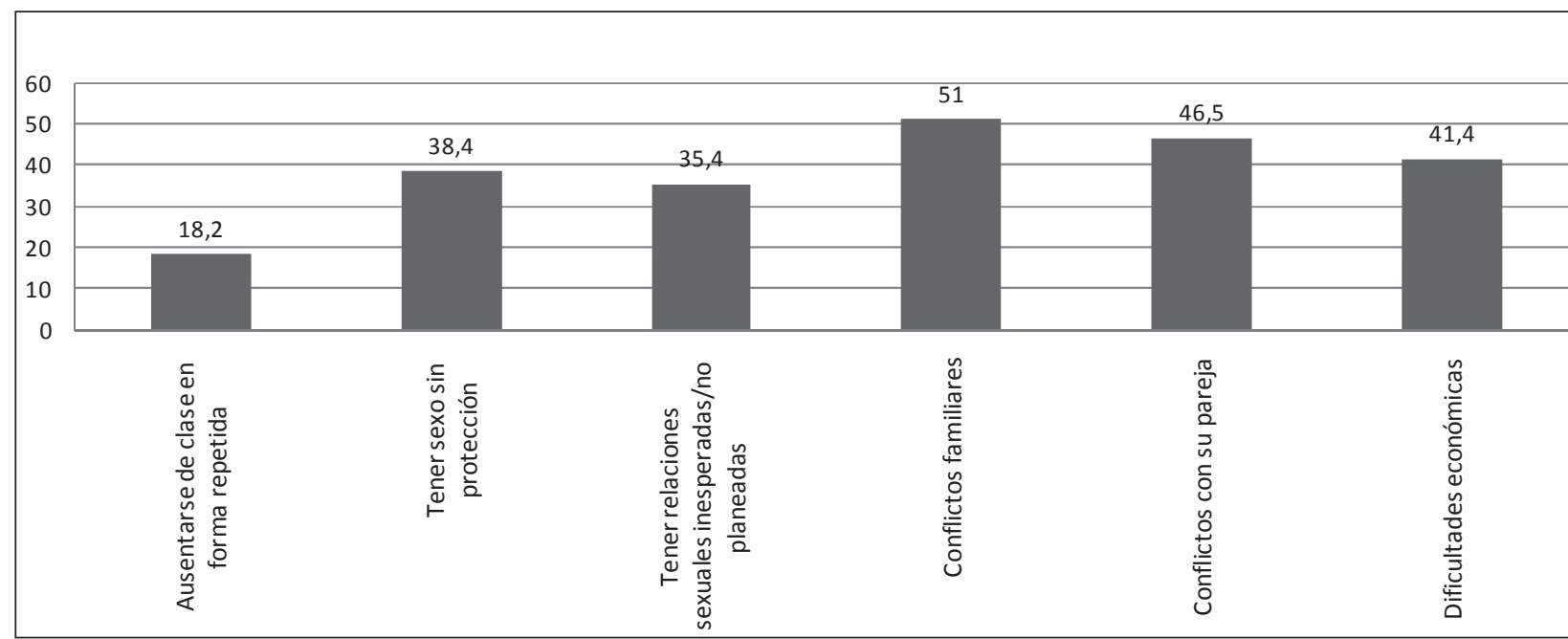

\section{DISCUSION}

El uso simultáneo de sustancias psicoactivas en estudiantes universitarios pertenecientes a programas académicos del área de la salud, es una situación bastante específica y poco estudiada; son pocos los estudios disponibles al respecto, especialmente en el contexto colombiano. Sin embargo se encuentran algunos estudios, ${ }^{8-11}$ referentes al uso simultáneo y no simultáneo de sustancias psicoactivas entre estudiantes universitarios y en algunos casos en estudiantes del área de la salud, en donde se hace alusión al uso simultáneo de sustancias psicoactivas, entre ellas el alcohol y el tabaco como combinación previa para el uso simultáneo de otras sustancias psicoactivas.

El Estudio Nacional de Consumo de Sustancias Psicoactivas en Colombia, 2008, y la Encuesta Nacional sobre Consumo de Sustancias Psicoactivas en Jóvenes entre 10 y 24 años, ${ }^{12}$ reportan como las sustancias psicoactivas de mayor consumo, el alcohol y el tabaco. En el estudio de 2008, el consumo de alcohol presenta las siguientes prevalencias: el $86.08 \%$ en la vida, el $61.18 \%$ en el último año y el $34.77 \%$ en el último mes. El consumo de tabaco presenta las siguientes prevalencias: el $44.49 \%$ en la vida, el $21.46 \%$ en el último año y el $17.06 \%$ en el último mes. Estas cifras son cercanas a los datos encontrados en nuestro estudio.

El patrón de uso simultáneo de alcohol y tabaco, podría constituirse en un factor de riesgo para la transición hacia el consumo de otras sustancias psicoactivas como marihuana y cocaína, lo cual es referido en otros estudios como "gateway model", lo que se describe como más frecuente en población joven. ${ }^{9}$

La información recolectada por nuestro estudio fue analizada en contexto desde la perspectiva de la triada: persona-sustancias psicoactivas-entorno ${ }^{13} \mathrm{y}$ de acuerdo a la Teoría del Aprendizaje Social ${ }^{14}$ y la Teoría Bio-psico-social. ${ }^{15}$ En relación al consumo simultaneo de alcohol y tabaco, se plantea la influencia de múltiples factores que se conjugan entre sí. En esta red, confluyen las características adictivas de estas sustancias psicoactivas, presentes en un ámbito de fácil adquisición, junto a las características de una persona joven que experimenta el proceso de transición del colegio a la universidad.

Esto se da en el marco de la interacción de numerosos factores constitucionales (genéticos y biológicos), sociales (actitudes, disponibilidad, presión) y psicológicos (historia de aprendizaje, tolerancia 
a la frustración, creencias). Este contexto nos hace plantear la necesidad de desarrollar estrategias de afrontamiento que den cuenta de las características del proceso del ciclo vital del joven universitario, las que facilitan una cultura de aceptación en cuanto al uso simultáneo de alcohol y tabaco.

Desde la teoría del aprendizaje social, se consideran dos aspectos, la "historia del aprendizaje social de la persona" y los "factores de modelado", caracterizados en el aprendizaje observacional ${ }^{14}$ Estos aspectos están representes en primera instancia, en el uso de las sustancias en el ambiente familiar por parte de los padres, hermanos y familiares, y en segunda instancia a través de las personas que hacen parte del ambiente social, como son los pares o grupos de iguales, además de su generalizada representación de uso en los anuncios publicitarios y en los medios de comunicación de masas. Por lo tanto, la historia personal del aprendizaje social y los factores de modelado, ejercen una importante influencia para la adquisición de la conducta, en donde se destaca como a través de esta se logra una mejor interacción con la gente, reducción del estrés social, así como bienestar físico y psicológico. ${ }^{14}$

En cuanto al lugar en donde se lleva a cabo el uso simultáneo de alcohol y tabaco, los sitios de reunión como las fiestas universitarias o las fiestas o reuniones sociales fuera del campus universitario, son escenarios de encuentro entre pares frecuentemente referidos en nuestra encuesta. En ellos, el ambiente seria propicio para el inicio en unos y el refuerzo en otros en torno al patrón de policonsumo simultáneo; allí se comparte y adquiere información, se imitan y también se establecen modelos como parte de los procesos de socialización y de aceptación entre los integrantes de un grupo en torno a un lenguaje común.

Esto resulta concordante con las denominadas representaciones sociales, ${ }^{16}$ que son el producto de los procesos de interacción social vivenciados por el estudiante universitario. En estos, el joven interpreta la realidad cotidiana desde la perspectiva de sus valores y creencias, y a su vez modela lo que considera favorable o desfavorable en el desarrollo de su comportamiento. Por ello el que se relacione el consumo de alcohol con experiencias placenteras como la percepción de cambios en la temperatura corporal y cambios sensoriales que se experimentan cuando el alcohol ha hecho efecto pero todavía no se ha consumido mucho, ${ }^{16}$ así como considerar su consumo como un facilitador social al permitirle desahogarse, al mismo tiempo que permite el dialogo.
Un antecedente de acuerdo a la teoría del aprendizaje social que hace parte del medio social y que actúa como estímulo para el uso simultáneo de alcohol y tabaco, se relaciona con las habilidades de afrontamiento; cuando estas son débiles y existe dificultad para manejar situaciones emocionales negativas o estresantes, este uso simultáneo aflora como estrategia de afrontamiento ante los problemas o situaciones identificadas como estresantes, reduciendo la ansiedad y la tensión e incrementando la sensación de manejo de la situación. Estas percepciones se podrían relacionar con el planteamiento en cuanto a las expectativas y creencias acerca de las drogas, como un antídoto para manejar la ansiedad y el estrés. ${ }^{14}$

La información generada en el estudio con relación a la vivencia de situaciones particulares y el uso simultáneo de alcohol y tabaco, se traduce en los resultados consecuencia de una acción, con una connotación negativa producto del resultado de procesos de afrontamiento que conducen a toma de decisiones traducidas en acciones riesgosas que generan malestar e incomodidad. Estos hallazgos se pueden relacionar con lo descrito en un estudio español, ${ }^{17}$ en donde se plantea que el consumo de sustancias psicoactivas puede asociarse a una variedad de consecuencias negativas, incluyendo un aumento en el riesgo de consumo de drogas años más tarde, fracaso escolar e irresponsabilidad, lo que puede poner al adolescente en riesgo de accidentes, violencia, relaciones sexuales no planificadas e inseguras, y suicidio. Asimismo, el consumo de sustancias psicoactivas se ha asociado consistentemente con una gama de otros comportamientos perjudiciales a la salud (por ejemplo, violencia no letal, como agresor o como víctima), así como innumerables padecimientos de salud.

Consideramos que el desarrollo de programas preventivos debe involucrar los aspectos mencionados, con el fin de incrementar las posibilidades para generar un alto impacto en el fenómeno de las drogas, situación que desafortunadamente sigue siendo un importante problema social, económico y político a nivel mundial.

\section{CONCLUSIONES}

Las características del medio social identificado en el estudio determinado por las variables (aprendizaje y lugar en donde se realiza el uso simultáneo de alcohol y tabaco, fuentes de estrés o ansiedad, razones para realizar este patrón de uso de sustancias psicoactivas y vivencia de situaciones relacionadas con el mismo), permite 
evidenciar la presencia de la triada de interacción conformada por el estudiante universitario, las sustancias psicoactivas y una serie de aspectos sociales como parte del entorno. Los componentes de esta triada interactúan mutuamente como eje de presión, generando características particulares, inherentes a la situación del uso simultáneo de alcohol y tabaco.

Las variables del medio social, conforman una serie de circunstancias que armonizan y se potencian entre sí permitiendo crear y recrear el imaginario en cuanto a que el uso simultáneo de alcohol y tabaco, se constituye en un importante facilitador social, en recurso para manejar las situaciones estresantes así como preámbulo para la vivencia de situaciones catalogadas como displacenteras y aún riesgosas y por lo tanto se legitima.

Es indiscutible como la disponibilidad, la inadecuada presión de pares o de grupo, así como la presión e influencia de los medios de comunicación con relación a las sustancias psicoactivas representadas en el alcohol y el tabaco, se constituyen en factores de riesgo que favorecen su uso simultáneo.

La información resultante del estudio se suma al conocimiento existente en la temática y permite contribuir en la planeación de programas de educación en salud al interior de las universidades. Para ello, creemos que el estudiante debe ser participe activo del proceso, incluyendo sus entornos familiar, universitario y su rol en la sociedad, a fin de empoderarse y lograr transformar el problema en una opción para el cambio, logrando así mayor bienestar en la construcción de su proyecto de vida.

\section{Limitaciones}

Los hallazgos de este estudio relacionados con el policonsumo simultaneo de alcohol y tabaco, reducen las posibilidades de conclusiones más generales en cuanto al fenómeno del policonsumo simultaneo de sustancias psicoactivas en estudiantes universitarios. Sin embargo, el hecho de ser la combinación referida con mayor frecuencia en estudios generales relacionados con este problema en la juventud, aumenta la utilidad de nuestros hallazgos. Los resultados obtenidos en este estudio no son generalizables a la población de jóvenes en general o a la de estudiantes universitarios, puesto que sólo se incluyeron estudiantes de primero y segundo años de carreras de Ciencias de la Salud en una universidad en Colombia.

\section{Recomendaciones}

Ampliar este estudio considerando un número mayor de estudiantes pertenecientes a otras facultades de la Universidad, así como en otras instituciones de educación superior del país para comprender mejor la dinámica de este fenómeno. Debido a que la evidencia apunta a que los estudiantes inician tempranamente el policonsumo simultáneo de sustancias psicoactivas, prácticamente tres o cuatro años antes de ingresar a la universidad, consideramos necesaria la realización de futuras investigaciones en estudiantes de educación secundaria, de tal forma que se pueda obtener una visión amplia en relación al ciclo vital, y de cómo los distintos cambios que se van presentando en su proceso de maduración influyen en la conducta del policonsumo. Realizar una investigación de diseño cualitativo con relación a la dinámica social en torno al policonsumo simultáneo de sustancias psicoactivas en estudiantes universitarios, a fin de direccionar el diseño de programas de salud de tipo preventivo.

\section{AGRADECIMIENTOS}

Al gobierno de Canadá/DFAIT, a la Organización de los Estados Americanos (OEA), Comisión Interamericana para el Control del Abuso de Drogas (CICAD), al Centre for Addiction and Mental Health (CAMH-Toronto/Canadá). Así mismo se agradece a los estudiantes de pregrado que participaron en el estudio, a las autoridades de la Universidad Participante, así como a todas las personas que colaboraron de forma directa o indirecta en la implementación del estudio. Un agradecimiento especial al Dr. Gustavo Mery, CAMH Reviewer, por su colaboración en la revisión y editoración final de todos los trabajos y informes del grupo III.

\section{REFERENCIAS}

1. Austria EUV. Se incrementa el uso de drogas en el mundo. ONU. El Siglo de Torreon; 2008.

2. CASA NCoAaSAaCU. Wasting the best and the brightest: substance abuse at america's colleges and universities. New York (US): Columbia University, National Center on Addiction and Substance Abuse. 2007.

3. McCabe SE, Cranford JA, Morales M, Young A. Simultaneous and concurrent poly-drug use of alcohol and prescription drugs: prevalence, correlates, and consequences. J Stud Alcohol. 2006 Jul; 67(4):529-37. 
4. Pillon S, O'Brien B, Chavez K. The relationship between drugs use and risk behaviors in brazilian university students. Rev Latino-am Enfermagem. 2005;13 (2):1169-76.

5. Bandura A. Pensamiento y acción. Englewood Cliffs, N.J. Barcelona (ES): Prentice Hall, Martínez Roca; 1986.

6. Huyse F, Lyons J, Stiefel F, Slates J, Jonge P, Latour C. Operationalizing the biopsychosocial model: the Intermed. Psychosomatics. 2001 Jan-Feb; 42(1):5-13.

7. Hernández R, Fernández C, Baptista P. Metodologia de la investigacion en ciencias sociales. $4^{\mathrm{a}} \mathrm{ed}$. Mexico D.F. (MX): McGraw-Hill; 2008.

8. Barrett S, Darredeau C, Pihl R. Patterns of simultaneous poly-substance use in drug using university students. Hum Psychopharmacol. 2006 Jun; 21(4):255-63.

9. Herrera-Vázquez M, Wagner FA, VelascoMondragón E, Borges G, Lazcano-Ponce E. Inicio en el consumo de alcohol y tabaco y transición a otras drogas en estudiantes de Morelos, México. Salud Pública Méx. 2004; 46(2):132-40.

10. Urrego D. Consumo de sustancias psicoactivas en estudiantes de especialidades médicas. Rev Salud Pública. 2002; Jan-Apr; 4(1):59-73.

11. Oliveira EB, Furegato ARF. El trabajo del estudiante de enfermería como un factor de riesgo para el consumo de alcohol y otras drogas. Rev Latino-am Enfermagem. 2008; 16(Esp):565-71.

12. Ministerio del Interior y de Justicia. Dirección Nacional de Estupefacientes. Colombia; 2009. [acceso 2009 Sept 20]. Disponible en: http:/ / scholar. google.com/scholar?hl=es\&q=encuesta+nacional + $\mathrm{de}+2001+\mathrm{en}+\mathrm{j} \% \mathrm{C} 3 \%$ B3venes+escolarizados+colom bianos + de $+10+a+24+a \% C 3 \%$ B1os.\&btnG $=$ Buscar\& $1 \mathrm{r}=\& a s \_y l o=\& a s \_v i s=0$

13. Sánchez Bravo-Villasante F. Sociología del consumo de alcohol en Asturias. Cuade Psiqa Comun. 2001;1(1):19-36.

14. Becoña E. Bases teóricas que sustentan los programas de prevención de drogas. Madrid (ES): Ministerio del Interior - Delegación del gobierno para el plan nacional sobre drogas; 2002.

15. Leal ER. Adolescentes y alcohol: la búsquedade sensaciones en un contexto social ycultural que fomenta el consumo. Apuntes de psicología. 2004;22:403-20.

16. Sierra DR, Pérez M, Pérez A, Núñez M. Representaciones sociales en jóvenes consumidores y no consumidores de sustancias psicoactivas. Colombia: Corporación Nuevos Rumbos; 2005.

17. García L, González MT, Egea E. Consumo de sustancias en chicas adolescentes. 2 ed. Islas Canarias: Fondo Social Europeo, Instituto Canario (ES) de La Mujer; 2008. 